submitted to Phys. Rev. B

\title{
Manifestation of Quantum Chaos in Electronic Band Structures
}

\author{
E. R. Mucciolo, R. B. Capaz, B. L. Altshuler, and J. D. Joannopoulos \\ Department of Physics, Massachusetts Institute of Technology, Cambridge, Massachusetts 02139, \\ $U S A$
}

(April 5, 1994)

\begin{abstract}
We use semiconductors as an example to show that quantum chaos manifests itself in the energy spectrum of crystals. We analyze the ab initio band structure of silicon and the tight-binding spectrum of the alloy $A l_{x} G a_{1-x} A s$, and show that some of their statistical properties obey the universal predictions of quantum chaos derived from the theory of random matrices. Also, the Bloch momenta are interpreted as external, tunable, parameters, acting on the reduced (unit cell) Hamiltonian, in close analogy to Aharonov-Bohm fluxes threading a torus. They are used in the investigation of the parametric autocorrelator of crystal velocities. We find that our results are in good agreement with the universal curves recently proposed by Simons and coworkers.
\end{abstract} PACS numbers: 05.45.+b, 71.90.+q, 73.20.Dx 


\section{INTRODUCTION}

The great success of band structure theory in providing a very accurate description of electronic properties of many materials is a well-established fact. Using few ingredients (like crystal structure and atomic number), one can obtain a great variety of material-dependent results (optical and transport properties, phonon spectrum, etc.) in very good agreement with the available experimental data. However, the implementation of a realistic band structure calculation is not a straightforward task and usually requires very complicated numerical algorithms. One is then led to ask whether there is some universal (materialindependent) behavior which may lay hidden in the apparent regularity of the bands. The universality should express some common characteristic of the underlying physical systems, in this case crystals. For instance, in the muffin-tin approximation, the motion of a valence electron inside a crystal can be pictured as that of a particle in a periodic billiard structure of smooth walls, whose classical dynamics is very likely to be chaotic [1,2]. If this simple analogy is valid, then one should be able to find in the electronic spectra of real crystals some of the universal signatures of quantum chaos [3, [1].

Quantum chaos occurs when a system exhibits chaotic dynamics in the classical limit. The best way to observe chaos is to break all continuous symmetries, so that the only constant of motion left is the total energy. In this case, we expect the energy spectrum to show some chaotic behavior, as well as some regularities (the so-called clean features). Chaos and discrete symmetries can coexist: one good example is the Sinai billiard [5]. However, discrete symmetries imply the partition of the eigenstates into classes and, consequently, the

existence of degeneracies in the spectrum. As we shall see below, lifting these degeneracies usually means removing part of the clean features, resulting in an enhancement of the universalities.

In a crystalline material the translation invariance allows us to reduce the problem to the study of the electron motion in a single unit cell with quasiperiodic boundary conditions. Denoting the periodic part of the single-electron wave function as $u_{\vec{k}}(\vec{r})$, the Schrödinger 
equation becomes

$$
H_{\vec{k}} u_{\vec{k}}(\vec{r})=E(\vec{k}) u_{\vec{k}}(\vec{r})
$$

where $\vec{k}$ is the Bloch momentum and the reduced Hamiltonian is

$$
H_{\vec{k}}=-\frac{\hbar^{2}}{2 m}(\nabla+i \vec{k})^{2}+V(\vec{r})
$$

The effective potential $V(\vec{r})$ has all the discrete symmetries (rotation, inversion, reflection, etc.) of the unit cell. The quasiperiodic boundary conditions provide a torus geometry to the unit cell and the Bloch momenta act as Aharonov-Bohm fluxes [6]. Notice that for $\vec{k}=0$ (at the so-called $\Gamma$ point) the Hamiltonian is real and time-reversal $(T)$ symmetric. Any $\vec{k} \neq 0$ internal to the Brillouin zone (i.e., which is not equivalent to $-\vec{k}$ by umklapping) breaks $T$. The potential removes the translational symmetry within the torus, making the problem chaotic.

Normally, one solves Eq. (11) for $\vec{k}$ varying along some special symmetry lines of the Brillouin zone and most bands calculated in this way will be degenerate. When we vary $\vec{k}$ away from the planes and lines of symmetry, all discrete symmetries of $H_{\vec{k}}$ are broken and all degeneracies are lifted, resulting in a "spaghetti" of bands (in this work we do not consider spin-orbit coupling or strong magnetic fields and Kramers degeneracy is always present). As an illustration, suppose we select a set of bands which are degenerate at the $\Gamma$ point and then follow them as we move in $\vec{k}$-space. We will notice that the bands split; eventually, some of them may come close together again, but if we do not cross any symmetry line, they will always "repel" (anticross) each other. Therefore, the Bloch momentum can be regarded as an external parameter which drives the system out of the partial integrability of the symmetry points and into a region where the spectrum is chaotic and bands are strongly correlated.

We expect the regions of the spectrum where chaos dominates to be short-ranged, since chaos and the consequent universality appear at very long time scales, translating into short energy scales. The clean features, on the other hand, are connected to the classical periodic 
orbits (short time scale) [2], which do not probe large portions of phase space: They will prevail in regions of the Brillouin zone close to the symmetry points, but can also be visible elsewhere.

We should contrast this view of chaos in crystals whose unit cells are fairly simple (few atoms per unit cell) with the more commonly discussed case of chaos in small disordered metallic grains (quantum dots). When there is no order or symmetry, but the system is small enough for individual energy levels to be distinguishable, it is very natural to study the level repulsion characteristic of quantum chaos and consider its implications to the thermodynamical properties of the system $[7,8]$. One can, for example, explore how much disorder is necessary to switch the system from nonchaotic to chaotic and how this crossover takes place [9]. Another idea which so far has only been developed theoretically is the study of chaos in arrays of identical disordered unit cells [1,10]. The translational invariance in these systems leads to the Hamiltonian of Eq. (2) and the same considerations we have just made for an elementary crystal should apply to the array of identical quantum dots. The only difference in this case is that the unit cell has no discrete symmetry and the spectrum is more likely to indicate strong chaotic dynamics. The clean features which persist in the band structure of the supercrystal will disappear after ensemble averaging.

The traditional diagnostic of quantum chaos derives from the original works of Wigner, Dyson, and Mehta on the theory of random matrices [11], which was initially designed for the study of statistical properties in Nuclear Physics. They have introduced most of the necessary mathematical tools: level spacing distributions, cluster functions, and several statistics, all focusing on level correlations. When a quantum system is subjected to an external perturbation, there are alternative ways to characterize chaoticity [3,12, 18]. The parametric correlation functions [15 [18] obtained by this approach are directly related to the transport properties of the system, hence bringing some important physical insight.

In this paper we show that an appropriate analysis of energy spectra obtained by band structure calculations indicates the unambiguous manifestation of quantum chaos in crystalline materials. Here we consider $S i$ as an example of a crystal which can be viewed as 
a quantum chaotic system with a particularly simple (diatomic) unit cell. Despite the fact that $T$ is broken for an internal $\vec{k}$, the space-inversion symmetry of the $S i$ crystal yields a false time-reversal violation [19] and the system is described by the Gaussian orthogonal ensemble (GOE) in the entire Brillouin zone. In contrast to Si, we also study the supercrystal formed by complex $A l_{x} G a_{1-x} A s$ cells. In this case chaos can be enhanced by increasing the amount of disorder through changing the concentration $x$. The lack of inversion symmetry of the unit cell causes $T$ to be quickly broken outside the $\Gamma$ point and therefore the ensemble is unitary (GUE).

\section{THE SILICON BAND STRUCTURE AND QUANTUM CHAOS}

In order to look for quantum chaos, we have to avoid doing the analysis of the spectrum at the $\Gamma$ point or at any symmetry point of the Brillouin zone. The effect of setting an internal $\vec{k} \neq 0$ is not just to lift the degeneracies caused by the point-symmetries of the unit cell. The Bloch momentum also serves to help increase the statistics by acting as a three-component external parameter. Once we have reached a region in the Brillouin zone where chaos is well developed and regularities are weak, we can tune $\vec{k}$ to generate a large number of spectra and hence facilitate the analysis.

As we have argued in the Introduction, even crystals with fairly simple unit cells should show quantum chaos. In order to verify this prediction, we have performed $a b$ initio electronic structure calculations of the Si energy bands. Our calculations were based on the local-density-functional and pseudopotential approximations. Details of the method are presented elsewhere [20]. A plane-wave cutoff of 15 Rydbergs was used in order to ensure a faithful description of the higher bands, which are most likely to show chaotic behavior than the low-lying ones.

The typical band dispersion for $S i$ is shown in Figure 1. The momentum varies from the $\Gamma$ point to the boundary of the Brillouin zone, passing by the center of mass $(\mathrm{CM})$ of the

irreducible part. Notice the band splitting for $\vec{k} \neq 0$. If the bands are truly uncorrelated, 
the distribution of band spacings $\epsilon$ is Poisson-like [3, [0]: $P(\epsilon) \propto e^{-\epsilon}$ (hereafter we will always express energies in units of the mean band spacing). If any correlation is present, the bands will tend to repel each other and $P(\epsilon \rightarrow 0) \rightarrow 0$. In Fig. 1 we see that several sets of bands which are degenerate at the $\Gamma$ point remain quite close together and do not seem to interact very much. This is caused by the presence of symmetry lines in the vicinity of the direction we have chosen to plot the bands. Indeed, this "memory effect" is very strong in the band structure of $S i$ and led us to concentrate our analysis at a small region around the CM, which is reasonably far from the $\Gamma$ point and other symmetry points.

It is clear from Eq. (2) that $H_{\vec{k}}$ is neither invariant under $T$, nor under space inversion $(P)$, in spite of the fact that the potential $V(\vec{r})=V(-\vec{r})$ for Si. In this case, however, $H_{\vec{k}}$ is invariant under the antiunitary combination $T P$ and this is sufficient to lead to GOE fluctuations, instead of GUE as one might naively expect [19].

The two most popular diagnostics of quantum chaos originally from random matrix theory (RMT) are the nearest-neighbor level spacing distribution, $P(\varepsilon)$, and the rigidity of the spectrum, the so-called $\Delta_{3}$ statistics. There is no expression for $P(\varepsilon)$ in closed form, but, as an excellent approximation, Wigner has proposed the surmise $P(\varepsilon) \propto \varepsilon^{\beta} e^{-c_{\beta} \varepsilon^{2}}$, where $\beta=1$ and $c_{1}=\pi / 4$ for GOE, and $\beta=2$ and $c_{2}=4 / \pi$ for GUE. The $\Delta_{3}$ statistics measures the variance of the number of levels found in an interval of length $L$ [1]:

$$
\Delta_{3}(L)=\frac{1}{L}\left\langle\operatorname{Min}_{a, b} \int_{\bar{E}-L / 2}^{\bar{E}+L / 2} d E[N(E)-a E+b]^{2}\right\rangle,
$$

where $N(E)$ is the number of energy levels below the energy $E$. The average indicated in Eq. (3) is performed over $\bar{E}$ (i.e., over nonoverlaping intervals between $\bar{E}-L / 2$ and $\bar{E}+L / 2$ ), but in our study it is also taken over points in $\vec{k}$-space. When the levels are completely uncorrelated (Poisson statistics), we have $\Delta_{3}(L)=\frac{L}{15}$. In the opposite limit of equally spaced levels, $\Delta_{3}(L)=\frac{1}{12}$. Sitting in between these two limits are the curves drawn from RMT, which have the $L \gg 1$ asymptotics [11]

$$
\Delta_{3}(L) \approx \frac{1}{\pi^{2}} \ln (L)-0.00696 \quad(\mathrm{GOE})
$$


and

$$
\Delta_{3}(L) \approx \frac{1}{2 \pi^{2}} \ln (L)+0.0590 \quad(\mathrm{GUE})
$$

In addition to these two quantities, RMT has also a prediction about the density-density (two-point) correlation function [11], here defined as

$$
R(\omega)=\langle\rho(\Omega+\omega) \rho(\Omega)\rangle-\langle\rho\rangle^{2}
$$

where $\rho(\Omega)=\sum_{n} \delta\left(\Omega-\varepsilon_{n}\right)$, and $\varepsilon_{n}=E_{n}$. The function $R(\omega)$ behaves differently depending on the particular ensemble: For GOE, it is linear close to $\omega=0$, and then monotonically saturates to 1 at around $\omega \approx 1$, whereas for GUE it starts as quadratic and then oscillates until it reaches saturation around the same values.

In Fig. 2a, 2b, and 2c we compare the statistical properties of the $S i$ spectrum with the RMT predictions. The data was extracted from a set of 80 high-energy eigenvalues corresponding to $343 \vec{k}$-points (a $7 \times 7 \times 7$ cube) around the CM. Notice the good agreement with the GOE result, in contrast to GUE. The deviation between the data points and the GOE curve for the $\Delta_{3}$ statistics at large $L$ (Fig. 2c) is expected because of the presence of clean features when we consider large portions of the spectrum.

The Bloch momentum can be used as an external, continuous, parameter, allowing us to evaluate "dynamical" universal correlation functions of the spectrum. For this purpose, one needs a scaling parameter, $\sqrt{C_{\mu \nu}(0)}$, which is related to the spectrum response to $\vec{k}$; namely,

$$
C_{\mu \nu}(0)=\left\langle\frac{\partial E_{n}(\vec{k})}{\partial k_{\mu}} \frac{\partial E_{n}(\vec{k})}{\partial k_{\nu}}\right\rangle,
$$

whre the average is performed over many energy bands (the index $n$ ), as well as over $\vec{k}$ points. Because in our study we dealt with correlation over small regions in $\vec{k}$-space, it was a good approximation to assume isotropy, i.e. $C_{\mu \nu}(0)=C(0)$ for all $\mu, \nu$. After performing the rescaling $x=\sqrt{C(0)} k_{\mu}$, where $\mu$ denotes some direction in $\vec{k}$-space, we evaluated one of the simplest correlation function one can study [16, 17], which is the autocorrelator of crystal velocities, 


$$
c(x)=\left\langle\frac{\partial \epsilon_{n}(\bar{x}+x)}{\partial \bar{x}} \frac{\partial \epsilon_{n}(\bar{x})}{\partial \bar{x}}\right\rangle,
$$

It is important to notice that in both Eq. (7) and (8) the bands $E_{n}(\vec{k})$ have to be corrected for any possible drift. The way we have proceeded was to estimate the local average drift $\bar{v}_{n}=\left\langle\partial E_{n}(\vec{k}) / \partial \vec{k}\right\rangle$ and then subtract it from the crystal velocities. We have found that $c(x)$ agrees reasonably with the universal form introduced in Ref. [17] for the pure GOE case (see Fig. 2d). The small, but visible, discrepancy is understandable: We have used only 7 points along each $\vec{k}$ direction and it is difficult to perform a good estimate of $C(0)$ and $\bar{v}_{n}$ for such a short interval.

\section{QUANTUM CHAOS IN THE $A l_{x} G a_{1-x} A s$ ALLOY}

In contrast to crystalline materials with simple unit cells, where the existence of symmetries causes partial integrability and the regularities in the spectrum tend to hide the underlying chaotic dynamics, disordered system are the ideal case to study. Classically, the motion of an electrons inside a disordered grain is that of a particle being repeatedly scattered by an irregular potential: The complete lack of symmetries will give rise to a strong chaotic motion. As a result, electronic disordered systems show very clear signatures of quantum chaos and the concept of universality is generally valid. The universal conductance fluctuations in mesoscopics systems [21] are a good example of a phenomenon related to the chaotic dynamics of the electron in the sample.

If there is one disadvantage of disordered systems over pure crystalline materials, it is the lack of Bloch momenta. From the theoretical view point, the natural way to solve this problem is to impose quasiperiodic boundary conditions to the disordered grain [10] and thus form a superlattice of identical complex unit cells. The band structure of this supercrystal can then be explored much in the same way as we did for $S i$.

In the analysis that follows we have chosen the widely studied $A l_{x} G a_{1-x} A s$ to demonstrate that it is indeed a good example of a quantum chaotic system and to illustrate the 
applicability of the parametric correlation functions to characterize quantum chaos. Alloys are a good example of weakly disordered systems when their components do not differ remarkably. If the sample is small enough (mesoscopic), the average level spacing can be resolved experimentally [22] and it makes sense to address the statistical properties of the spectrum. The absence of discrete symmetries in the unit cell guarantees that there are no degeneracies in the spectrum, although some regularities may occurs and they are usually connected to the nonuniversal features carried by the isolated components of the alloy.

Our study of the $A l_{x} G a_{1-x} A s$ supercrystals is based on a semiempirical tight-binding method, with matrix elements taken from the $s p^{3} s^{*}$ parametrization suggested by Vogl [23]. Several ensemble realizations of a 216-atom basic cluster were independently generated and solved (the electron wave function was subjected to quasiperiodic boundary conditions). For each realization, $A l$ and $G a$ atoms were randomly distributed in the group-III sublattice according to the aimed alloy composition. Alloy properties are calculated as ensemble averages for each composition. This method has been successfully used in the study of gap properties of the random $A l_{x} G a_{1-x} A s$ alloy [24].

We begin by calculating the level spacing distribution of $A l_{x} G a_{1-x} A s$ at the $\Gamma$ point (hence the boundary conditions are periodic) for three different compositions, namely $x \approx$ $0.1,0.3$, and 0.5 (note that for $0<x<1$ the spectrum is completely nondegenerate). The results for the level spacing distribution are shown in Fig. 3. For a given composition $x$, the averaging is done over high-energy levels and over 20 realizations. Notice that as the disorder increases, we move from a Poisson-like law to a GOE-like, Wigner-Dyson, distribution (remember that at the $\Gamma$ point there is no $T$ breaking). Another indication of the crossover between weakly to strongly correlated energy levels can be seen in Fig. 4, where we have plotted the $\Delta_{3}$ statistics for the three alloy compositions.

Next we introduce phases to the boundary conditions to obtain the dispersion of the bands with the Bloch momenta. In Fig. 5 we show a typical set of bands at the high-energy part of the spectrum for a composition $x \approx 0.5$. As for the case of $S i$, we restrict our analysis to the region surrounding the CM. The clean features now are fewer and usually 
related to regularities found in the band structure of $A l A s$ and GaAs [25]; one can be seen in the upper part of Fig. 5.

Focusing on the high-energy bands and averaging over 9 realizations, we obtain the results shown in Fig. 6. Notice the excellent agreement with the GUE predictions. For any Bloch momentum outside the $\Gamma$ point, $T$ is fully broken and since the unit cell is not invariant under space inversion, we naively expect the statistics to be GUE. In fact, this issue is more subtle. There is a continuous change from GOE to GUE as we move away from the $\Gamma$ point [4] and the typical range of this crossover will depend on the specific system under study. After the proper rescaling, the crossover should be universal and at least two theoretical investigations based on the supersymmetric technique have demonstrated this point [26,27]. We have, as yet, not performed an analysis of this crossover for the band structure of the $A l_{x} G a_{1-x} A s$ supercrystal. Nevertheless, we stress that our results show that at the CM the system has already attained the GUE limit.

It is worth to remark the good agreement between the data for $c(x)$ and the curve obtained in Ref. [17] through numerical simulations (see Fig. 6d). The deviation at $x \sim 1$ happens because nonuniversal features dominate the correlation at large distances. We mention that, in principle, we should be able to see the universal behavior of the response function predicted in Ref. 10 by calculating suitable matrix elements of chaotic eigenstates. We leave this subject for future investigation.

\section{CONCLUSIONS}

In summary, we have demonstrated that quantum chaos is present in the band structures of $S i$ and of the $A l_{x} G a_{1-x} A s$ supercrystal. We have argued that this should hold true for any crystal because valence electrons exhibit classical chaotic dynamics at the level of the unit cell. The Bloch momentum can be viewed as an external parameter which can be tuned to break the discrete symmetries of the unit cell and revel the quantum chaos hidden in the regularity of the band structure. Any crystal whose unit cell is invariant under space 
inversion should be described by the GOE statistics. Violation of the inversion symmetry combined with a deviation from the $\Gamma$ point drives the system to GUE statistics. The parametric correlation functions were shown to be a good diagnostic of quantum chaos, not only for system with complex unit cells, like the supercrystal of $A l_{x} G a_{1-x} A s$, but also for the diatomic unit cell case of Si. The important result of our work is that there exists universality in band structures. The implications of this property to experiments may, however, be limited. For instance, the optical properties of crystals are usually defined by few, low-lying, bands close to the Fermi level, which do not show very strong quantum chaos. One way to increase the complexity of low energy levels is to consider real crystals with polyatomic unit cells.

\section{Acknowledgments}

The authors are greatful to Ben Simons for many illuminating discussions throughout the evolution of this work. We also thank Nobuhiko Taniguchi, Anton Andreev, and Tomás Arias for useful conversations, and Belita Koiller for providing the tight-binding code. E.R.M. and R.B.C. would like to acknowledge the financial support of Conselho Nacional de Desenvolvi-

mento Científico e Tecnológico (CNPq, Brazil). This work was supported by the Joint Services Electronic Program no. DAAL 03-89-0001. 


\section{REFERENCES}

[1] R. B. Laughlin, Nucl. Phys. B (Proc. Suppl.) 2, 213 (1987).

[2] M. C. Gutzwiller, Chaos in Classical and Quantum Mechanics (Springer-Verlag, New York, 1990).

[3] F. Haake, Quantum Signatures of Chaos (Springer-Verlag, Berlin, 1991).

[4] O. Bohigas in Chaos and Quantum Physics, edited by M.-J. Giannoni, A. Voros, and J. Zinn-Justin (North-Holland, Amsterdam, 1991), p. 87.

[5] Ya. G. Sinai, Ups. Mat. Nauk. 25, 137 (1970) [Russ. Math. Surv. 25, 137 (1970)].

[6] M. V. Berry and M. Robnik, J. Phys. A 19, 649 (1986).

[7] L. P. Gor'kov and G. M. Eliashberg, Zh. Eksp. Teor. Fiz. 48, 1407 (1965) [Sov. Phys. JETP 21, 940 (1965)].

[8] B. Mühlschlegel, in Chaos and Quantum Physics, edited by M.-J. Giannoni, A. Voros, and J. Zinn-Justin (North-Holland, Amsterdam, 1991), p. 727.

[9] O. Bohigas, M.-J. Giannoni, and C. Schmidt, Quantum Chaos and Statistical Nuclear Physics, edited by T. H. Seligman and N. Nishioka (Springer-Verlag, Berlin, 1986), p. 18.

[10] N. Taniguchi and B. L. Altshuler, Phys. Rev. Lett. 71, 4031 (1993).

[11] M. L. Mehta, Random Matrices, 2nd. ed. (Academic Press, San Diego, CA, 1991).

[12] M. Wilkinson, J. Phys. A 21, 4021 (1988); Phys. Rev. A 41, 4645 (1990).

[13] P. Gaspard, S. A. Rice, H. J. Mikeska, and K. Nakamura, Phys. Rev. B 42, 4015 (1990).

[14] J. Zakrzewski and D. Delande, Phys. Rev. E 47, 1650 (1993).

[15] J. Goldberg, U. Smilansky, M. V. Berry, W. Schweizer, G. Wunner, and G. Zeller, Nonlinearity 4, 1 (1991). 
[16] A. Szafer and B. L. Altshuler, Phys. Rev. Lett. 70, 587 (1993).

[17] B. D. Simons, A. Szafer, and B. L. Altshuler, JETP Lett. 57, 276 (1993); B. D. Simons and B. L. Altshuler, Phys. Rev. Lett. 70, 4063 (1993); Phys. Rev. B 48, 5422 (1993).

[18] C. W. J. Beenakker, Phys. Rev. Lett. 70, 4126 (1993); C. W. J. Beenakker and B. Rejaei (preprint).

[19] M. Robnik and M. V. Berry, J. Phys. A 19, 669 (1986).

[20] M. C. Payne, M. P. Teter, D. C. Allan, T. A. Arias, and J. D. Joannopoulos, Rev. Mod. Phys. 64, 1045 (1992).

[21] There is an extensive literature on this topic. For a recent review, see Mesoscopic Phenomena in Solids, edited by B. L. Altshuler, P. A. Lee, and R. A. Webb (North-Holland, Amsterdam, 1991).

[22] M. A. Kastner, Rev. Mod. Phys. 64, 849 (1992).

[23] P. Vogl, H. P. Hjalmarson, and J. D. Dow, J. Phys. Chem. Solids 44, 365 (1983).

[24] R. B. Capaz, G. C. de Araújo, B. Koiller, and J. P. von der Weid, J. Appl. Phys. 74, 5531 (1993); R. B. Capaz, J. P. von der Weid, and B. Koiller, Appl. Phys. Lett. 60, 704 (1992).

[25] The band folding caused by the contraction of the Brillouin zone makes it hard to associate a specific clean feature found in the band structure of the $A l_{x} G a_{1-x} A s$ supercrystal to its counterpart in the band structures of $A l A s$ or GaAs.

[26] A. Altland, S. Iida, and K. B. Efetov, J. Phys. A 26, 3545 (1993).

[27] N. Taniguchi, A. Hashimoto, B. D. Simons, and B. L. Altshuler (unpublished). 


\section{FIGURES}

1. The band structure for $S i$. The Bloch momentum runs from the $\Gamma$ point $(\vec{k}=0)$ to the boundary of the Brillouin zone, passing through the center of mass of the irreducible part $(\mathrm{CM})$. The scale is such that $k=1$ at the $\mathrm{CM}$.

2. Statistical properties of 90 high-energy bands of $S i$ around the CM point. The solid and dashed lines are the GOE and GUE predictions, respectively. Figure (a) is the band spacing distribution; (b) is the autocorrelator of density of states (rescaled to $\langle\rho\rangle=1$ ); (c) is the $\Delta_{3}$ statistics; (d) is the crystal velocity correlation function $c(x)$. Error bars are of the order of the data point symbol size or smaller, whenever not indicated. For all curves the average was taken over the bands and $\vec{k}$-points. The sets of points in (d) differ by the number

of bands used in the estimate of the local average drift, $\left\langle\partial E_{n}(\vec{k}) / \partial \vec{k}\right\rangle$. They correspond to: 7 (circles), 9 (triangles), and 11 bands (diamonds).

3. The level spacing distribution involving about 150 high-energy eigenstates at $\Gamma$ in the $A l_{x} G a_{1-x} A s$ supercrystal for concentrations: (a) $x \approx 0.1$; (b) $x \approx 0.3$; (c) $x \approx 0.5$. The average was taken over 20 realizations of a given concentration. The circles indicate the data and the solid and dashed lines the GOE and Poisson statistics, respectively.

4. The $\Delta_{3}$ statistics for the same set of levels and realizations of Fig. 3, following the same conventions.

5. Typical band dispersion around the CM point for the supercrystal of $A l_{x} G a_{1-x} A s$ cells $(x=0.5)$. The region presented here is particularly free of strong clean features, although a weak one can be seen at the top of the spectrum. The scale of the momentum axis has arbitrary units and is centered at the CM. 
6. Statistical properties for the high-energy bands of the $A l_{x} G a_{1-x} A s$ supercrystal around the CM point at $x=0.5$. The circles indicate the data and the solid and dashed lines the GUE and GOE predictions, respectively (error bars are typically smaller than the circles). Figure (a) is the band spacing distribution; (b) is the autocorrelator of density of states; (c) is the $\Delta_{3}$ statistics; (d) is the crystal velocity correlation function $c(x)$. For (a), (b) and (c) the average was taken over bands and ensemble (9 realizations); for (d) the average was only over ensembles. The data for $c(x)$ was found to be insensitive to the way the average drift was estimated. 